\title{
Edge Texture Based CBIR using Row Mean of Transformed Column Gradient Image
}

\author{
Dr.H.B.Kekre ${ }^{1}$, \\ Sudeep Thepade ${ }^{2}$ \\ ${ }^{1}$ Sr.Professor, ${ }^{2}$ Asso. Prof., \\ MPSTME, SVKM's NMIMS \\ University, Mumbai
}

\author{
Priyadarshini Mukherjee, \\ Miti Kakaiya, \\ B.Tech Student, \\ MPSTME, SVKM's NMIMS \\ University, Mumbai
}

\author{
Shobhit Wadhwa, \\ Satyajit Singh, \\ B.Tech Student, \\ MPSTME, SVKM's NMIMS \\ University, Mumbai
}

\begin{abstract}
The paper discusses novel image retrieval methods based on edge texture of images extracted using gradient operators and slope magnitude technique with image transforms. Twenty eight variations of proposed image retrieval techniques using seven image transforms and four gradient operators like Roberts, Sobel, Prewitt and Canny are considered here. The proposed image retrieval techniques are tested on generic image database with 1000 images spread across 11 categories. In all 55 queries (5 from each category) are fired on the image database. The average precision and recall of all queries are computed and considered for performance analysis. The various 'Mask-Shape-Transform' CBIR techniques [1] are compared with each other and each of the proposed 'MaskShape-Transform' CBIR methods. The 'Mask-ShapeTransform' is found to be better than the 'Mask-Shape' Technique. In all Roberts-Kekre-Transform based CBIR gives best performance followed by Sobel-Slant-Transform and Sobel-Hartley-Transform.
\end{abstract}

\section{Keywords}

CBIR, Edge Texture, Slope Magnitude, Transforms, Cosine, Sine, Haar, Hartley, Kekre, Slant, Walsh.

\section{INTRODUCTION}

Information retrieval (IR) is the science of searching for documents, for information within documents, and for metadata about documents, as well as that of searching relational databases and the World Wide Web. There is overlap in the usage of the terms data retrieval, document retrieval, information retrieval, and text retrieval, but each also has its own body of literature, theory and technologies. $\mathrm{IR}$ is interdisciplinary, based on computer science, mathematics, cognitive psychology, linguistics, statistics, and physics. Web search engines are the most visible IR applications. Images do have giant share in this information being stored and retrieved, making image retrieval one of the key research field in IR. Content Based image retrieval (CBIR) is the area where searching is done using image content. The images are very rich in the content like color, texture and shape information present in them [2]. Retrieving images based on color similarity is achieved by computing a color histogram for each image that identifies the proportion of pixels within an image holding specific values (that humans express as colors) [11]. Color searches usually involve comparing color histograms [16], color averages [19], BTC [20] and other methods. Texture measures look for visual patterns in images and how they are spatially defined [14]. The identification of specific textures in an image is achieved primarily by modeling texture as a two-dimensional gray level variation, GLCM [10], vector quantization codebooks [6], image transforms [7]. Shape does not refer to the shape of an image but to the shape of a particular region that is being sought out. Shapes will often be determined first applying segmentation or edge detection to an image [12]. Other methods use shape filters to identify given shapes of an image [14]. In some case accurate shape detection will require human intervention because methods like segmentation are very difficult to completely automate [15]. Here the paper discusses shape extraction using edge detection masks like Sobel, Roberts, Prewitt and Canny gradient operators [13]. The edge texture is generated using row mean of transformed columns of the extracted edge image with slope magnitude method.

\section{IMAGE TRANSFORMS}

Image transforms [17,18] can be simple arithmetic operations on images or complex mathematical operations which convert images from one representation to another. In the paper seven image transforms are used. The list of these image transforms is given below

$\begin{array}{ll}\text { - } & \text { Discrete Cosine Transform(DCT) [8,9] } \\ \text { - } & \text { Discrete Sine Transform (DST) } \\ \text { - } & \text { Haar Transform } \\ \text { - } & \text { Hartley Transform } \\ \text { - } & \text { Kekre's Transform [4] } \\ \text { - } & \text { Slant Transform } \\ \text { - } & \text { Walsh Transform [5] }\end{array}$



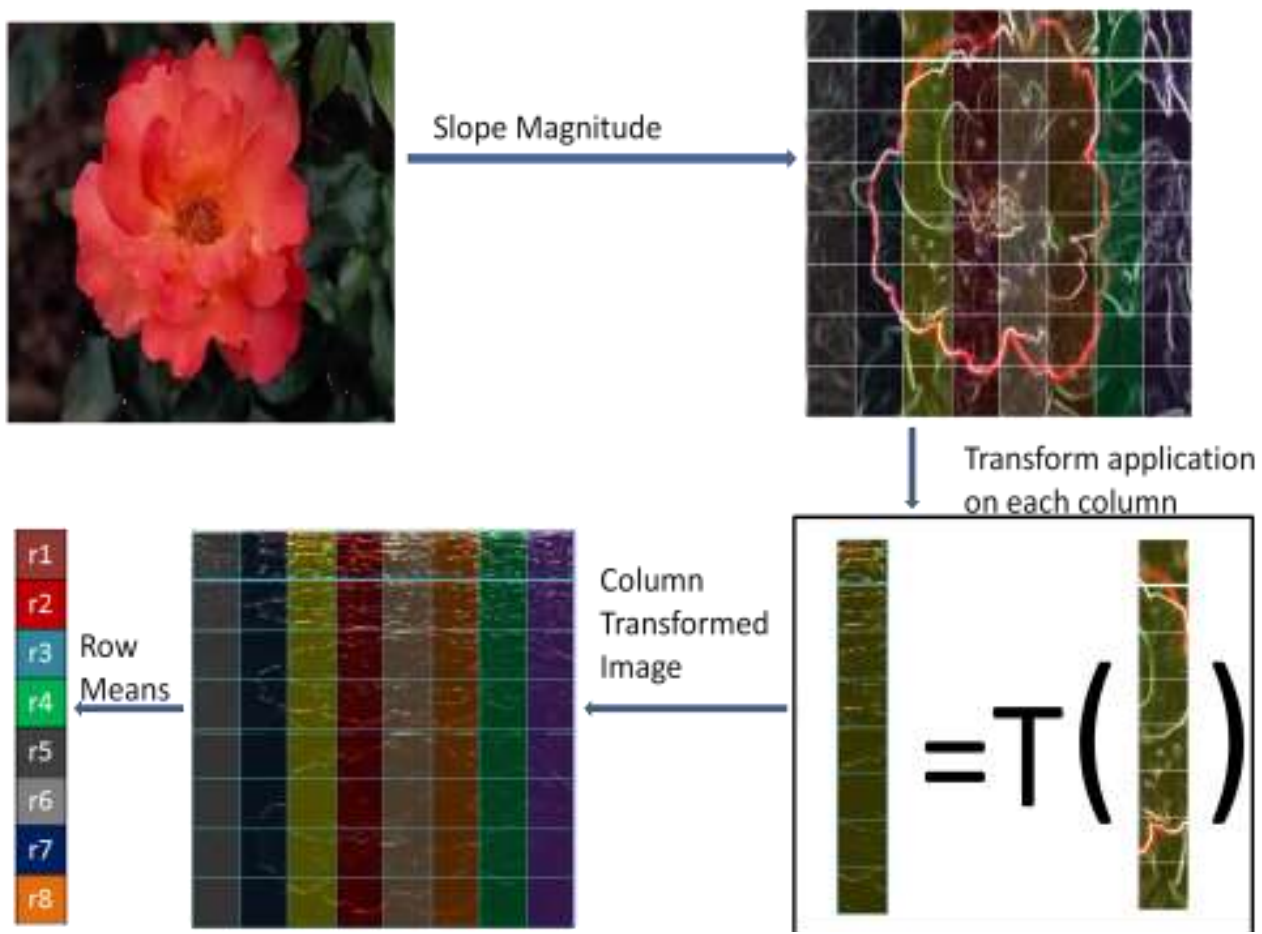

Figure 1: Edge Texture Extraction using Gradient Operators and Image Transforms

\section{EDGE EXTRACTION}

The problem with edge extraction using gradient operators is detection of edges in only either horizontal or vertical directions, as the gradient operators take the first order derivative of image in either vertical or horizontal direction only. Shape feature extraction in image retrieval requires the extracted edges to be connected in order to reflect the boundaries of objects present in the image. Slope magnitude method [1] is used along with the gradient operators (Sobel, Prewitt, Robert and Canny)[1] to extract the shape features in form of connected boundaries. The process of applying the slope magnitude method is given as follows. First the image needs to be convolved with the Gx mask to get the $\mathrm{x}$ gradient and Gy mask to get the $\mathrm{y}$ gradient of the image. Then the individual squares of both these gradients are taken. Square root of addition of the two squared terms gives the extracted connected edges from the image as given in equation 1.

$$
G=\sqrt{G_{x}^{2}+G_{y}^{2}}
$$

\section{EDGE BASED CBIR TECHNIQUE}

In 'Mask Shape' CBIR [1] the shape feature vector is formed by applying slope magnitude method on Gradient of image in vertical and horizontal directions. This feature vector is considered for finding the similarity of query image with database images. The problem of this technique is that all the images in the database must have same dimensions as that of query image. The selection of gradient operators like Robert, sobel, prewitt and canny results into four variations of 'Mask Shape' based image retrieval.

\section{PROPOSED CBIR TECHNIQUES}

Here firstly, the edges of the image are extracted by applying slope magnitude method on gradients of the image in vertical and horizontal directions and then the transforms is applied on each column of obtained 'Mask Shape'. The row mean of these transformed column Mask-Shape image is obtained and treated as image signature for CBIR. The row means for individual $R, G$ and $B$ planes forms the feature vector. This has been shown in figure 1 . Total 24 variations of proposed CBIR technique are possible by using four gradient operators namely Sobel, Roberts, Prewitt and Canny with seven orthogonal image transforms namely DCT, DST, Haar, Hartley, Walsh, Kekre and Slant.

\section{IMPLEMENTATION}

The discussed image retrieval methods are implemented using MATLAB 7.0 on Intel Core 2 Duo processor T8100(2.1 GHz) with $2 \mathrm{~GB}$ of RAM. To check the performance of proposed technique a database of 1000 variable sized images spread across 11 categories has been used [3]. Five queries were selected from each category of images. Mean Squared Error (MSE) is used as similarity measure for comparing the query image with all the images in the image database. Let Vpi and Vqi be the feature vectors of image 'P' and Query image ' $Q$ ' respectively 
with size ' $n$ ', then the MSE can be given as shown in equation 2 .

$$
M S E=\sum_{i=1}^{n}(V p i-V q i)^{2}
$$

To assess the retrieval effectiveness, we have used the precision and recall as statistical comparison parameters for our proposed technique of CBIR. The standard definitions of these two measures are given by following equations.

$$
\begin{gathered}
\text { Precision }=\frac{\text { Number of relevant images retrieved }}{\text { Total } \text { number of } \text { images retrieved }} \\
\text { Recall }=\frac{\text { Number of relevant images retrieved }}{\text { Total number of relevant images in database }}
\end{gathered}
$$

\section{RESULTS AND DISCUSSIONS}

The graphs of average precision and recall values plotted against the number of retrieved images obtained from the various techniques are shown as figure 2 to figure 6 . In figure 2 the performances of basic mask-shape-CBIR techniques for various gradient operators are compared. Here canny operator gives better image retrieval as indicated by higher precision and recall values.

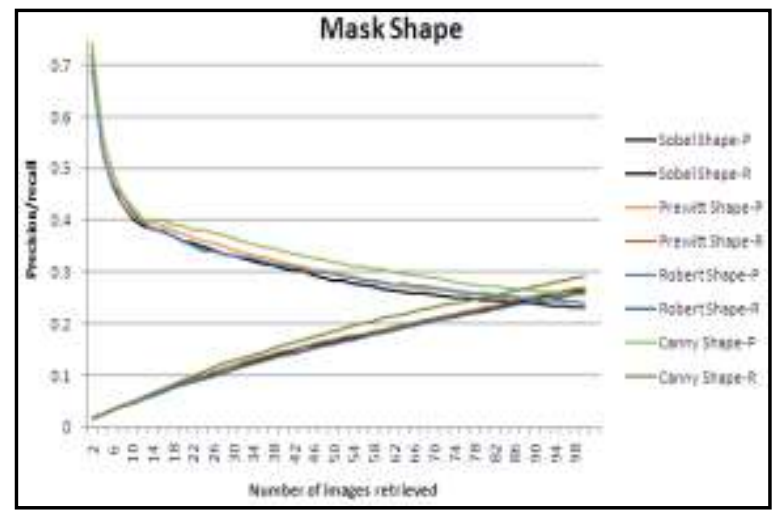

Figure 2: Mask Shape technique for all gradient operators [1]

The precision and recall values for Canny gradient operator with proposed CBIR techniques is plotted in figure 3. Here all image transforms except Kekre transform gives same and better performance. Figure 4 shows performance the average precision and recall values plotted against the number of retrieved images for proposed CBIR technique using Prewitt gradient operator. Here Kekre transform gives best performance as compared to other image transforms.

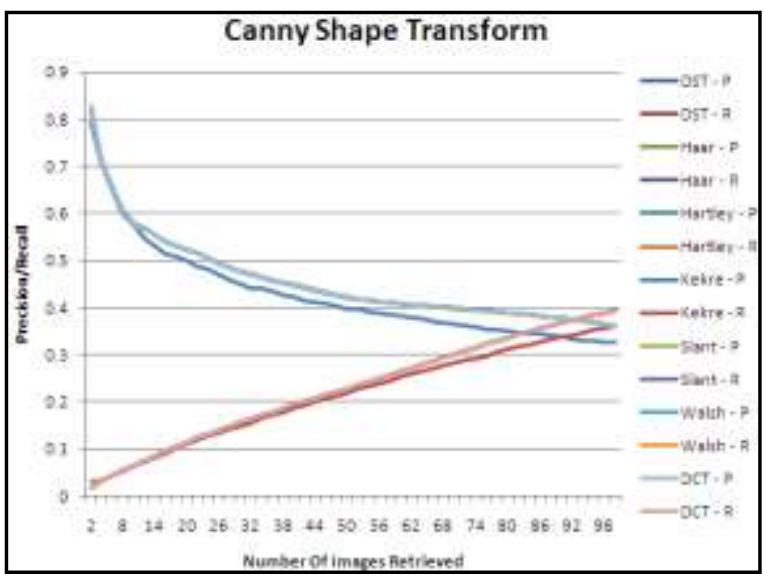

Figure 3: Canny gradient for all transforms

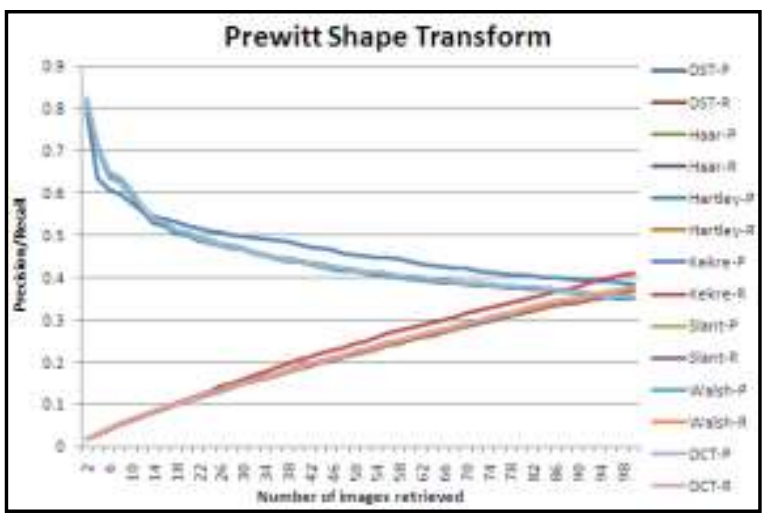

Figure 4: Prewitt gradient for all transforms

The precision and recall values for Robert gradient operator with proposed CBIR techniques is plotted in figure 5. Here DCT gives the best image retrieval with highest precision and recall values. Figure 6 shows performance the average precision and recall values plotted against the number of retrieved images for proposed CBIR technique using Sobel gradient operator. Here Slant and Hartley transforms give best performance as compared to other image transforms.

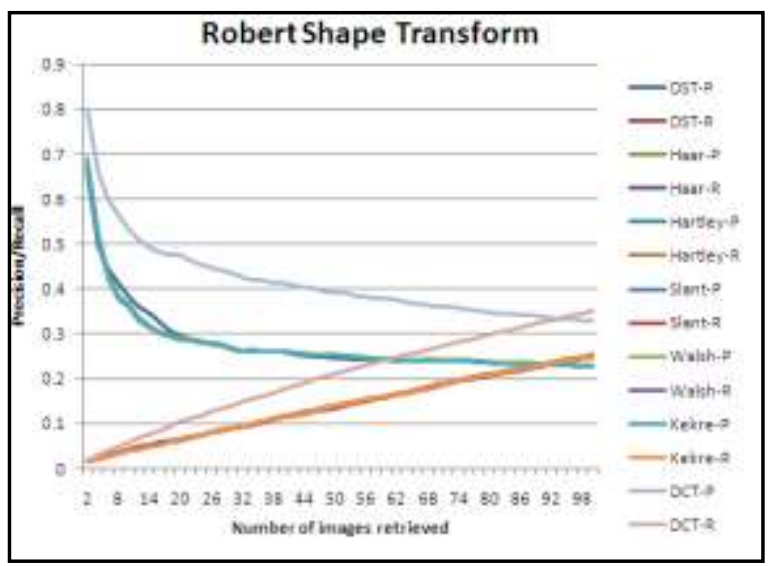

Figure 5: Robert gradient for all transforms 


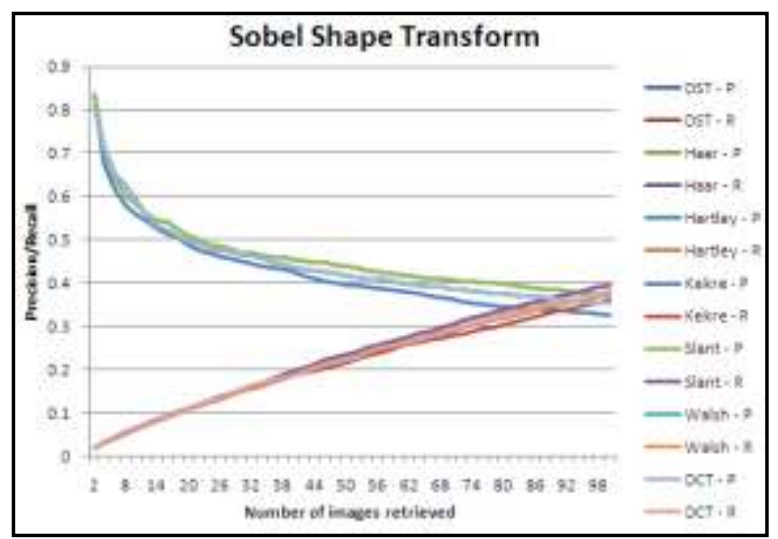

Figure 6: Sobel gradient for all transforms

The performance comparison of using different gradient operators for each transform is given in figure 7 . Here the crossover point of average precision and recall for each image transform with the considered gradient operators used to extract edge are plotted. The height of this crossover point plays important role in performance comparison of various CBIR techniques. More height of precision-recall crossover point indicates better performance. In all proposed CBIR methods for Canny, Sobel and Prewitt gradient operators performance is improved for all transforms as compared to the ealier discussed Mask-Shape-CBIR methods [1].

Figure 8 gives performance comparison of individual gradient operators for used image transforms. In Prewitt operator Kekre transform shows best performance. In Sobel operator best performance is indicated by Hartley and Slant transforms. DCT proves to be best for Robert operator. In Canny all transform except Kekre indicates best performance.

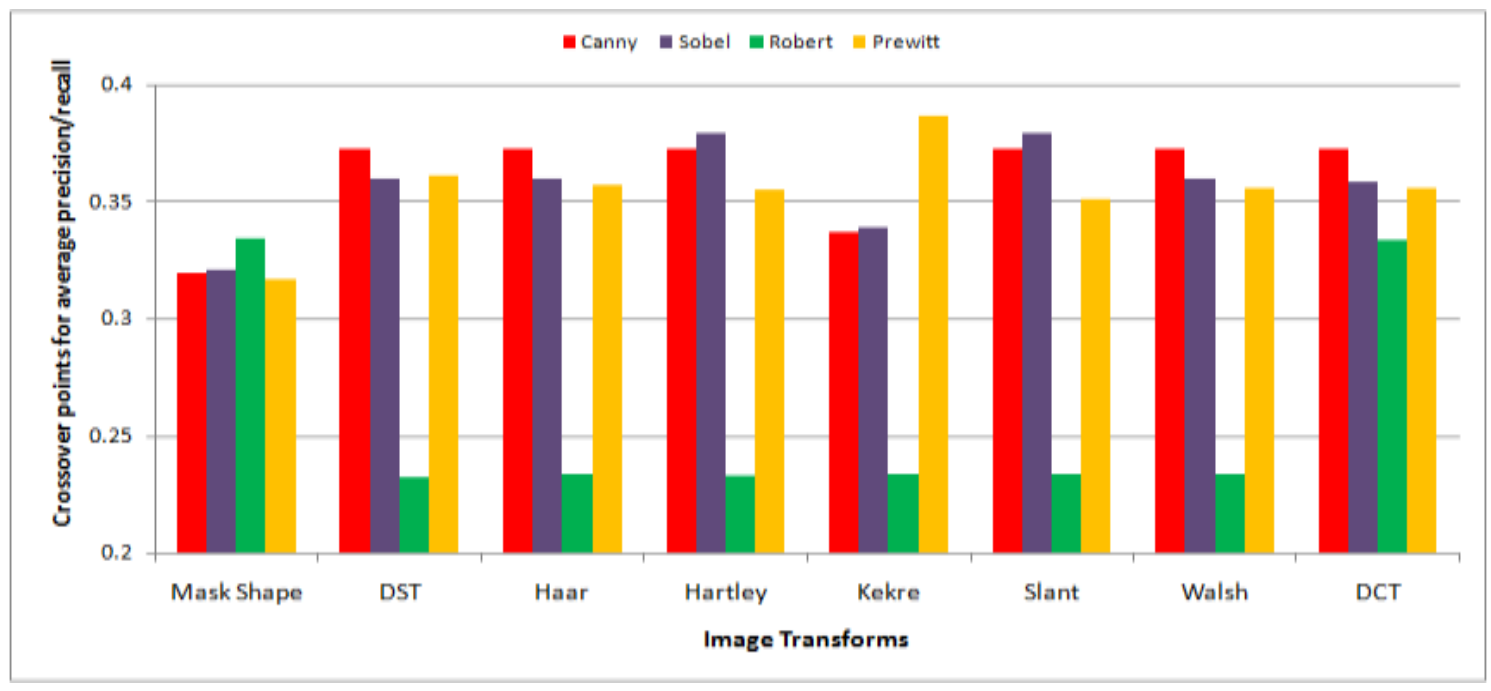

Figure 7: Performance Comparison of Gradient Operators for Individual Image Transforms

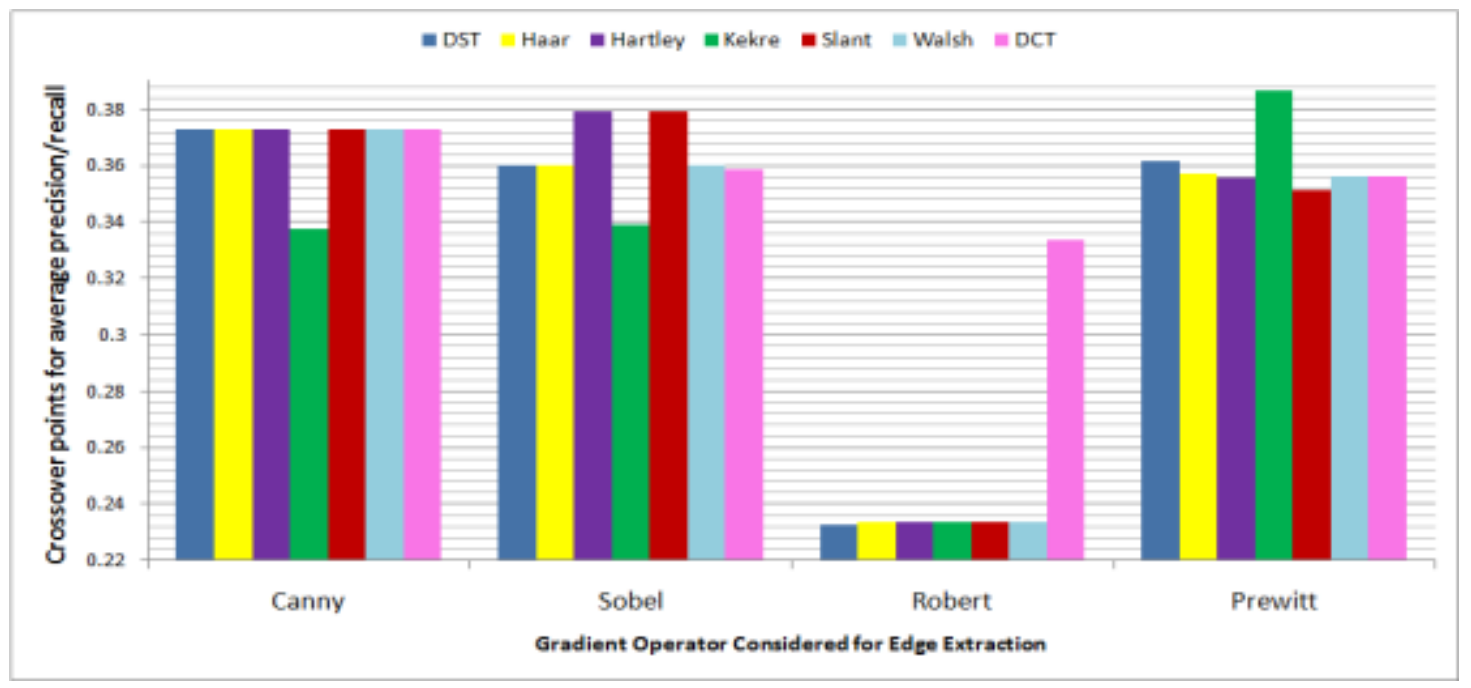

Figure 8: Performance Comparison of Image Transforms for individual Gradient Operators 
Among all 24 variations of newly proposed CBIR method, the Prewitt-Kekre-Transform based technique proves to be the best. The next performance is given by Sobel-SlantTransform CBIR and Sobel-Hartley-Transform CBIR.

\section{CONCLUSION}

Image retrieval is one of important thirst area for researchers from image processing and information technology domain. The desire of better and faster image retrieval is still not satisfied. The paper proposes the novel and better CBIR technique using edge texture extracted as row mean of transformed column gradient image with slope magnitude method. Using four gradient operators and seven image transforms, set of 24 variations of proposed CBIR methods is tested on the image database of 1000 images spread across 11 image classes. The experimental results have proved the proposed CBIR technique to be better than the Mash-Shape-CBIR given in [1] for all considered gradient operators except Roberts. In all the proposed CBIR techniques with Prewitt operator and Kekre transform gives the best performance as indicated by highest precision and recall values. The second best performance is given in case of using Sobel gradient operator with Slant or Hartley image transform for proposed CBIR method.

\section{REFERENCES}

[1]. Dr.H.B.Kekre, Sudeep D. Thepade, Priyadarshini Mukherjee, Shobhit Wadhwa, Miti Kakaiya, Satyajit Singh, "Image Retrieval with Shape Features Extracted using Gradient Operators and Slope Magnitude Technique with BTC", International Journal of Computer Applications, September 2010 issue.

[2]. Dr.H.B.Kekre, Sudeep D. Thepade, "Rendering Futuristic Image Retrieval System", National Conference on Enhancements in Computer, Communication and Information Technology, EC2IT-2009, 20-21 Mar 2009, K.J.Somaiya College of Engineering, Vidyavihar, Mumbai-77.

[3]. Image database http://wang.ist.psu.edu/docs/related/Image.orig (Last referred on 23 Sept 2008)

[4]. Dr.H.B.Kekre, Sudeep D. Thepade, Archana Athawale, Anant Shah, Prathmesh Verlekar, Suraj Shirke,"Energy Compaction and Image Splitting for Image Retrieval using Kekre Transform over Row and Column Feature Vectors", International Journal of Computer Science and Network Security (IJCSNS),Volume:10, Number 1, January 2010, (ISSN: 1738-7906) Available at www.IJCSNS.org.

[5]. Dr.H.B.Kekre, Sudeep D. Thepade, "Image Retrieval using Color-Texture Features Extracted from Walshlet Pyramid", ICGST International Journal on Graphics, Vision and Image Processing (GVIP), Volume 10, Issue I, Feb.2010, pp.9-18,
Available

online www.icgst.com/gvip/Volume10/Issue1/P115093887 6.html

[6]. Dr.H.B.Kekre, Tanuja Sarode, Sudeep D. Thepade, "Color-Texture Feature based Image Retrieval using DCT applied on Kekre's Median Codebook", International Journal on Imaging (IJI), Volume 2, Number A09, Autumn 2009,pp. 55-65. Available online at www.ceser.res.in/iji.html

[7]. Dr.H.B.Kekre, Sudeep D. Thepade, "Image Retrieval using Non-Involutional Orthogonal Kekre's Transform", International Journal of Multidisciplinary Research and Advances in Engineering (IJMRAE), Ascent Publication House, 2009, Volume 1, No.I, pp 189-203, 2009. Abstract available online at www.ascent-journals.com

[8]. Dr.H.B.Kekre, Sudeep D. Thepade, "Improving the Performance of Image Retrieval using Partial Coefficients of Transformed Image", International Journal of Information Retrieval, Serials Publications, Volume 2, Issue 1, 2009, pp. 72-79

[9]. Dr.H.B.Kekre, Sudeep D. Thepade, Archana Athawale, Anant Shah, Prathmesh Verlekar, Suraj Shirke, "Performance Evaluation of Image Retrieval using Energy Compaction and Image Tiling over DCT Row Mean and DCT Column Mean", Springer-International Conference on Contours of Computing Technology (Thinkquest-2010), Babasaheb Gawde Institute of Technology, Mumbai, 13-14 March 2010, The paper will be uploaded on online Springerlink.

[10]. Dr.H.B.Kekre, Tanuja K. Sarode, Sudeep D. Thepade, Vaishali Suryavanshi,"Improved Texture Feature Based Image Retrieval using Kekre's Fast Codebook Generation Algorithm", SpringerInternational Conference on Contours of Computing Technology (Thinkquest-2010), Babasaheb Gawde Institute of Technology, Mumbai, 13-14 March 2010, The paper will be uploaded on online Springerlink.

[11]. Hirata K. and Kato T. "Query by visual example content-based image retrieval”, In Proc. Of Third International Conference on Extending Database Technology, EDBT'92, 1992, pp 56-71.

[12]. Sagarmay Deb, Yanchun Zhang, "An Overview of Content Based Image Retrieval Techniques," Technical Report, University of Southern Queensland.

[13]. Rafael C. Gonzalez, Richard E. Woods, "Digital Image Processing". Chapter 10, pg 599-607. Published by Pearson Education, Inc. 2005.

[14]. William I. Grosky, "Image Retrieval - Existing Techniques, Content-Based (CBIR) Systems" Department of Computer and Information Science, University of Michigan-Dearborn, Dearborn, MI, USA,http://encyclopedia.jrank.org/articles/pages/67 
63/Image-Retrieval.html\#ixzz0130drFVs, referred on 9 March 2010

[15]. Bill Green, "Canny Edge Detection Tutorial”, 2002. http://www.pages.drexel.edu/ weg22/can_tut.html, referred on 9 March 2010

[16]. John Eakins, Margaret Graham, "Content Based Image Retrieval", Chatpter 5.6, pg 36-40, University of Northrumbia at New Castle, October 1999

[17]. Dr.H.B.Kekre, Sudeep D. Thepade, Akshay Maloo, "Performance Comparison of Image Retrieval Techniques using Wavelet Pyramids of Walsh, Haar and Kekre Transforms", International Journal of Computer Applications (IJCA) Volume 4, Number 10, August 2010 Edition, pp 1-8, http://www.ijcaonline.org/archives/volume4/numbe r10/866-1216

[18]. Dr.H.B.Kekre, Sudeep D. Thepade, Akshay Maloo, "Performance Comparison of Image Retrieval Using Fractional Coefficients of Transformed Image Using DCT, Walsh, Haar and Kekre's Transform", CSC International Journal of Image Processing (IJIP), Volume 4, Issue 2, pp 142-157, Computer Science Journals, CSC Press, www.cscjournals.org

[19]. Dr.H.B.Kekre, Sudeep D. Thepade, Varun K. Banura, "Amelioration of Colour Averaging Based Image Retrieval Techniques using Even and Odd parts of Images", International Journal of Engineering Science and Technology (IJEST), Volume 2, Issue 9, Sept. 2010. pp. (ISSN: 09755462) Available online at http://www.ijest.info.

[20]. Dr.H.B.Kekre, Sudeep D. Thepade, "Boosting Block Truncation Coding using Kekre's LUV Color Space for Image Retrieval”, WASET International Journal of Electrical, Computer and System Engineering (IJECSE), Volume 2, No.3, Summer 2008. Available online at www.waset.org/ijecse/v2/v2-3-23.pdf

\section{AUTHOR BIOGRAPHIES}

Dr. H. B. Kekre has received B.E. (Hons.) in Telecomm. Engineering. from Jabalpur University in 1958, M.Tech (Industrial Electronics) from IIT Bombay in 1960, M.S.Engg. (Electrical Engg.) from University of Ottawa in 1965 and Ph.D. (System Identification) from IIT Bombay in $1970 \mathrm{He}$ has worked as Faculty of Electrical Engg. and then HOD Computer Science and Engg. at IIT Bombay. For 13 years he was working as a professor and head in the Department of Computer Engg. at Thadomal Shahani Engineering. College, Mumbai. Now he is Senior Professor at MPSTME, SVKM's NMIMS University. He has guided 17 Ph.Ds, more than 100 M.E./M.Tech and several B.E./B.Tech projects. His areas of interest are Digital Signal processing, Image Processing and Computer Networking. He has more than 320 papers in National / International Conferences and Journals to his credit. He was Senior Member of IEEE. Presently He is Fellow of IETE and Life Member of ISTE Recently ten students working under his guidance have received best paper awards and two have been conferred Ph.D. degree of SVKM's NMIMS University. Currently 10 research scholars are pursuing Ph.D. program under his guidance

Sudeep D. Thepade has Received B.E.(Computer) degree from North Maharashtra University with Distinction in 2003. M.E. in Computer Engineering from University of Mumbai in 2008 with Distinction, currently pursuing Ph.D. from SVKM's NMIMS University, Mumbai. He has about than 07 years of experience in teaching and industry. He was Lecturer in Dept. of Information Technology at Thadomal Shahani Engineering College, Bandra(w), Mumbai for nearly 04 years. Currently working as Associate Professor in Computer Engineering at Mukesh Patel School of Technology Management and Engineering, SVKM's NMIMS University, Vile Parle(w), Mumbai, INDIA. He is member of International Association of Engineers (IAENG) and International Association of Computer Science and Information Technology (IACSIT), Singapore. He is reviewer for many international journals and in the international advisory panel for many international conferences. He has worked as member of International Advisory Committee for many International Conferences. His areas of interest are Image Processing and Computer Networks. He has about 80 papers in National/International Conferences/Journals to his credit with a Best Paper Award at Int. Conference SSPCCIN2008, Second Best Paper Award at ThinkQuest-2009 National Level faculty paper presentation competition and Best Paper Award at Springer Int. Conf. ICCCT-2010

Shobhit Wadhwa is pursuing a B.Tech degree in Information Technology from MPSTME, SVKM's NMIMS University, Mumbai, India. His areas of interest lie in image processing and information systems development. He is also a member of the IEEE committee of his college.

Satyajit Singh is pursuing a B.Tech degree in Information Technology from MPSTME, SVKM's NMIMS University, Mumbai,India. His areas of interest lie in the fields of Image processing and Wireless technologies.

Priyadarshini Mukherjee is pursuing a B.Tech degree in Information Technology from MPSTME, SVKM's NMIMS University, Mumbai. Her interests lie in the fields of image processing and website development.

Miti Kakaiya is pursuing a B.Tech degree in Information Technology from MPSTME, SVKM's NMIMS University, Mumbai. Her interests lie in the field of Image processing and website designing. 